Nikolaus Seiler Jacques-Philippe Moulinoux
ADRESSES

N. Seiler: docteur ès sciences, professeur associé. J.Ph. Moulinoux: docteur en médecine, docteur ès sciences, praticien universitaire, praticien hospitalier, directeur de l'URA Cnrs 1529. Groupe de recherche en thérapeutique anticancéreuse, Cnrs URA 1529 affilièe à l'Inserm, Laboratoire de biologie cellulaire, Faculté de Médecine de Rennes I, 2, avenue du Professeur-Léon-Bernard, 35043 Rennes Cedex, France.

\title{
Les polyamines présentent-elles un intérêt dans le traitement du cancer?
}

Le métabolisme des polyamines étant fortement activé dans les cellules cancéreuses, l'inhibition de la synthèse de ces molécules a constitué une cible pour l'industrie pharmaceutique. Mais une succession d'échecs a rendu sceptique quant à cette voie thérapeutique. En fait, l'inhibition de l'anabolisme intracellulaire des polyamines doit être couplée à une réduction de leur absorption intestinale pour empêcher la progression tumorale. Associés aux médicaments anticancéreux usuels, les inhibiteurs de la synthèse des polyamines potentialisent leurs effets. Récemment des analogues de polyamines mimant les polyamines naturelles sans pour autant être capables d'en assumer les fonctions, se sont révélés actifs vis-à-vis de la croissance tumorale in vivo. Enfin, du fait d'un système de transport transmembranaire spécifique, certains analogues de polyamines s'accumulent préférentiellement dans le tissu tumoral. Cette notion a suscité la synthèse de molécules cytotoxiques couplées à une polyamine, ce qui devrait améliorer leur ciblage tumoral et corrélativement une réduction de leurs effets secondaires toxiques. Il n'existe donc plus d'argument objectif permettant d'écarter le métabolisme des polyamines du champ des recherches à visée anticancéreuse.

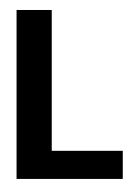

'importance biologique d'amines telles que la putrescine, la spermidine et la spermine (figure 1), communément appelées «polyamines », provient du fait qu'il s'agit d'ingrédients universels de toute cellule eucaryote.

Les polyamines remplissent de multiples fonctions dans les cellules eucaryotes. La plupart d'entre elles sont dues aux interactions électrostatiques qu'engagent ces polycations organiques avec des sites anioniques présents au niveau des acides nucléiques (ADN, ARN), des protéines, des membranes cellulaires. Les polyamines se comportent ainsi comme des cations métalliques tels le $\mathrm{Na}^{+}$, le $\mathrm{K}^{+}$, le $\mathrm{Ca}^{2+}$, le $\mathrm{Mg}^{2+} \ldots$ mais à la différence de ces derniers, dont la source est par définition extracellulaire, non 


\section{RÉFÉRENCES}

1. Bachrach U, Heimer YM. The physiology of polyamines. Boca Raton, USA: CRC Press, 1988.

2. Marton LJ, Pegg AE. Polyamines as targets for therapeutic intervention. Annu Rev Pharmacol Toxicol 1995; 35 : 55-91.

3. McCann PP, Pegg AE, Sjoerdsma A. Inhibition of polyamine metabolism. Orlando, USA: Academic Press, 1987.

4. Levin VA, Prados MD, Yung WKA, Gleason MJ, Ictech S, Malec M. Treatment of recurrent gliomas with eflornithine. J Natl Cancer Inst 1992; 84: 1432-7.

5. Seiler N, Dezeure F. Polyamine transport in mammalian cells. Int J Biochem 1990; 22 : 211-8.

6. Sarhan S, Knödgen B, Seiler N. The gastrointestinal tract as polyamine source for tumor growth. Anticancer Res 1989; 9: 21524.

7. Bardocz S. The role of dietary polyamimes. Eur J Clin Nutr 1993; 47: 683-90.

8. Seiler N, Sarhan S, Mamont P, Casara P, Danzin C. Some biological consequences of S-adenosylmethionine decarboxylase inhibition by MDL 73.811. Life Chem Rep 1991; 9 : 51-162.

9. Byers TL, Wiest L, Wechter RS, Pegg AE. Effect of chronic 5'-( (Z)-4-amino-butenyl)méthylamino-5'-deoxyadenosine (AbeAdo) treatment on polyamine and elF-5A metabolism in AbeAdo-sensitive and resistant L1210 murine leukemia cells. Biochem J 1993: 115-21.

10. Regenass U, Mett H, Stanek J, Mueller M, Kramer D, Porter CW. A new S-adenosylmethionine decarboxylase inhibitor with broad spectrum antiproliferative and antitumor activity. Cancer Res 1994; 54: 3210-7.

11. Ask A, Persson L, Heby O. Increased survival of L1210 leukemic mice by prevention of the utilization of extracellular polyamines. Studies using a polyamine-uptake mutant, antibiotics and a polyamine deficient diet. Cancer Lett 1992; 66 : 29-34.

12. Khan NA, Quemener V, Moulinoux $\mathrm{J}-\mathrm{Ph}$, Characterization of polyamine transport pathways. In: Carter $\mathrm{C}$, ed The neuropharmacology of polyamines. London, UK: Academic Press, 1994: 37-60.

13. Bergeron RJ, Neims AH, McManis JS, Hawthorne TR, Vinson JRT, Bortell R, Ingeno M.J. Synthetic polyamine analogues as antineoplastics. J Med Chem 1988; 31: 1183-

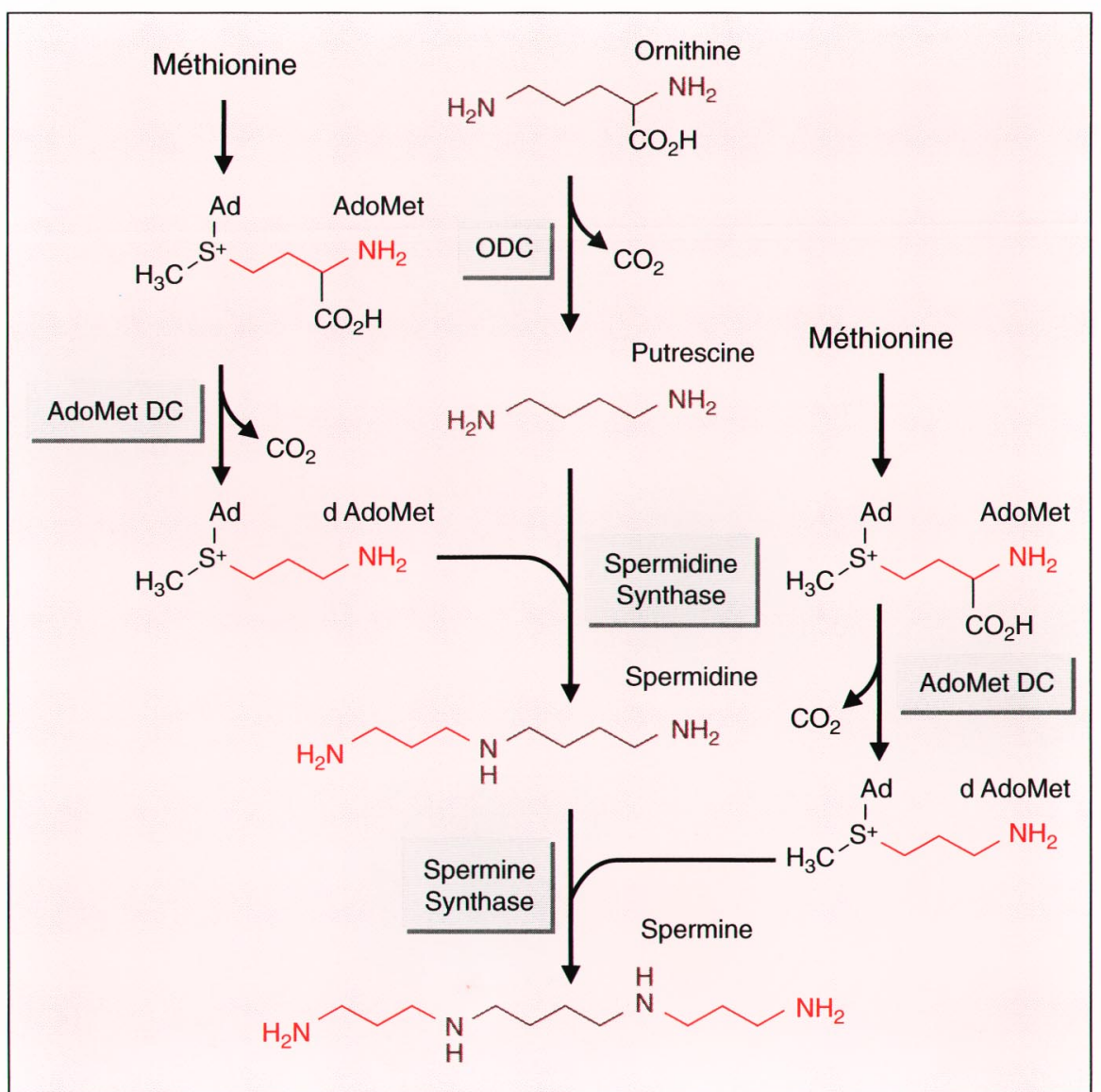

Figure 1. Métabolisme intracellulaire des polyamines. L'ornithine décarboxylase $(O D C)$ et la S-adénosylméthionine décarboxylase (AdoMetDC) constituent les deux enzymes-clés de l'anabolisme des polyamines. AdoMet: $S$-adénosylméthionine; dAdoMet: S-adénosylméthionine décarboxylée.

seulement les polyamines sont synthétisées «à la demande» par les cellules, mais ces molécules peuvent de plus engager des liaisons amides avec des protéines pour modifier certaines de leurs propriétés physiques ou biologiques [1]. Ainsi, l'hypusine qui est un acide aminé dérivé de la spermidine, est un constituant de cofacteur d'inititation de la synthèse de peptides chez l'eucaryote (IF5A).

L'anabolisme des polyamines étant accru dans les cellules cancéreuses et son inhibition s'accompagnant systématiquement d'un arrêt de croissance de ces dernières, ce métabolisme a été considéré comme indispensable à la prolifération cancéreuse et, à ce titre, est devenu une cible pour la synthèse de molécules à visée anticancéreuse [2]. Parmi celles-ci, la 2(difluorométhyl)ornithine (DFMO) (figure 2) s'est révélée être de loin la plus prometteuse. In vitro, la DFMO est capable d'inactiver de manière spécifique l'ornithine décarboxylase (ODC) - c'est-à-dire la biosynthèse intracellulaire de la putrescine - et d'inhiber la prolifération des cellules cancéreuses [3] ; l'arrêt de la prolifération cellulaire provoquée par inhibition de l'ODC est par ailleurs levée dans tous les cas par ajout de putrescine ou de spermidine au milieu de culture, ce qui montre que l'effet antiprolifératif de la DFMO est effectivement dû à la réduction des concentrations intracellulaires de putrescine et de spermidine.

L'administration de DFMO à des animaux porteurs de tumeurs, puis à des patients atteints de cancer, ne s'est pourtant accompagnée que d'effets antitumoraux très modestes $[3,4]$.

A l'optimisme initial succéda ainsi le scepticisme quant à l'impact thérapeutique de l'inhibition du métabolisme des polyamines dans le cancer. 
Nous estimons que ce scepticisme n'est plus actuellement de mise. En effet, comme nous l'exposons plus loin, notre meilleure compréhension du métabolisme des polyamines, d'une part, et, d'autre part, les bases expérimentales précliniques dont nous disposons maintenant, nous confortent dans l'idée que ce métabolisme de prolifération peut désormais constituer un outil thérapeutique de première importance pour le traitement du cancer.

\section{Pour quelles raisons I'inactivation de l'ornithine décarboxylase ne s'accompagne-t-elle pas in vivo d'une inhibition de la croissance tumorale?}

L'ornithine décarboxylase (ODC) et la S-adénosylméthionine décarboxylase (AdoMetDC) constituent les deux enzymes-clés de l'anabolisme des polyamines (figure 1) [1]. Cultivées en présence de DFMO - ou d'un autre inhibiteur spécifique de l'ODC - les cellules ont des concentrations de putrescine et de spermidine très diminuées (figure 3); la plupart d'entre elles deviennent rapidement incapables d'entrer en phase $S$, ce qui peut être en relation avec une réduction de la biosynthèse de protéines essentielles à la traversée du cycle cellulaire. L'inhibition de l'ODC provoquée par l'administration de DFMO s'accompagne de trois effets indésirables au niveau du métabolisme des polyamines: (1) l'induction de l'AdoMetDC et l'accumulation intracellulaire consécutive de S-adénosylméthionine décarboxylée (dAdoMet): dans ces conditions la spermine continue d'être synthétisée à partir d'un pool résiduel intracellulaire de spermidine, ce qui explique dans une large mesure l'absence de réduction des concentrations cellulaires de spermine (figure 3) ; (2) si le niveau d'inhibition de l'ODC est insuffisant, par exemple du fait d'une quantité insuffisante de DFMO accessible aux cellules, on constate alors, non seulement un accroissement intracellulaire de l'activité, mais encore de la quantité de protéine ODC; (3) les cellules

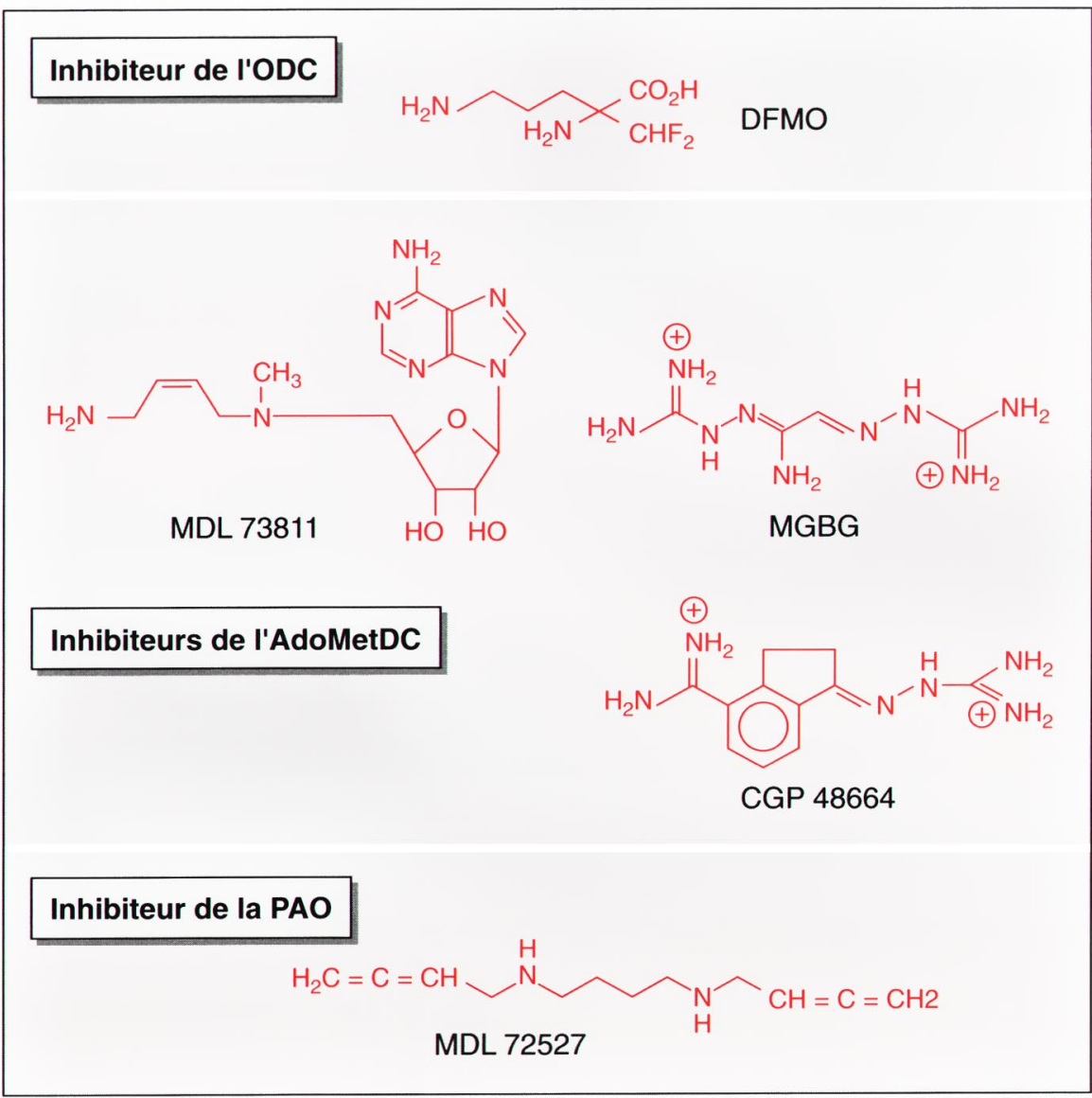

Figure 2. Inhibiteurs de l'anabolisme des polyamines. ODC: ornithine décarboxylase; AdoMetDC: S-adénosylméthionine décarboxylase; $P A O$ : polyamine oxydase ; DFMO: 2-(difluorométhyl)ornithine; MDL 73.811: 5'-((Z)-4-amino-butenyl)méthylamino-5'-déoxyadénosine ; MGBG : méthylglyoxal-bis(guanyl)hydrazone.

carencées en polyamines accroissent leur capacité de transport actif transmembranaire vis-à-vis des polyamines présentes dans le milieu environnant (sang, LCR, etc.) [5].

Il faut signaler que la spermine captée en milieu extracellulaire peut également favoriser la prolifération des cellules carencées en putrescine et en spermidine; ces dernières possèdent, en effet, la capacité de transformer la spermine en spermidine, et la spermidine en putrescine, via la rétroconversion oxydative des polyamines (figure 4), une voie métabolique qui constitue par conséquent une source endogène de putrescine et de spermidine utilisable par les cellules cancéreuses [1]. Dans un système fermé, ce qui est le cas d'une culture cellulaire, le fait que la quantité de polyamines présente dans le milieu de culture soit quantitative- ment négligeable et qu'il soit techniquement possible de maintenir une concentration suffisante en inhibiteur de l'ODC permet d'expliquer que l'inhibition de l'anabolisme des polyamines puisse provoquer l'inhibition totale de la prolifération cellulaire cancéreuse. Mais, à la différence d'une culture cellulaire, un organisme constitue un système ouvert et ses cellules ont, de fait, accès à des polyamines extracellulaires, tout particulièrement à celles véhiculées dans le sang, comme cela a été montré par injection de polyamines radiomarquées; ces polyamines circulantes ont, de plus, tendance à s'accumuler préférentiellement dans les cellules tumorales. Les polyamines présentes en milieu extracellulaire sont issues de sources exogènes et endogènes. S'il est actuellement encore difficile de spécifier chacune d'entre elles, un 

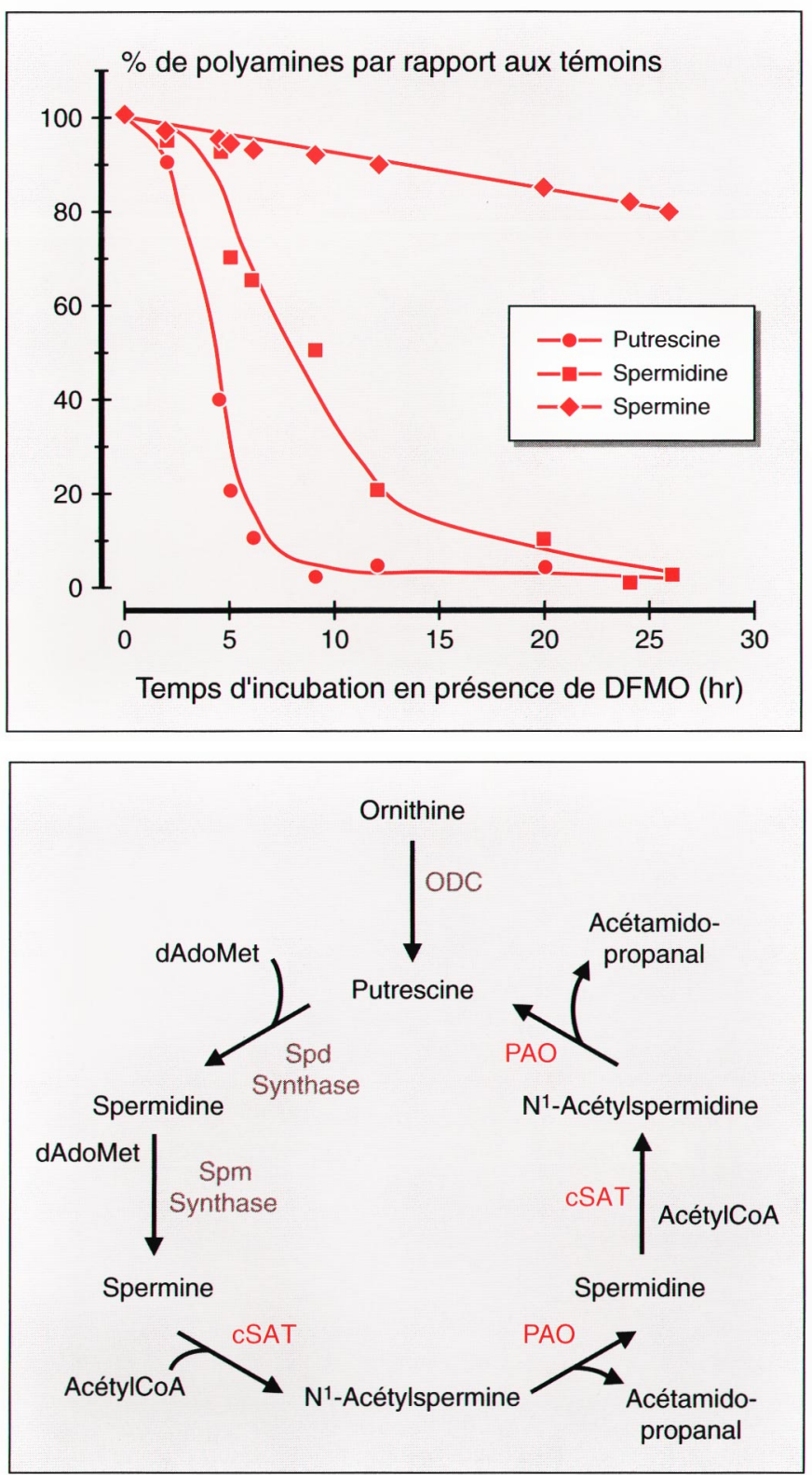

Figure 3. Concentrations en polyamines dans des hépatocytes cancéreux cultivés en présence de DFMO (5mM) (d'après [44]). Cultivées en présence de DFMO, les cellules ont des concentrations de putrescine et de spermidine très diminuées. L'inhibition de l'ODC provoquée par l'administration de DFMO s'accompagne de l'induction de l'AdoMetDC et de l'accumulation intracellulaire consécutive de S-adénosylméthionine décarboxylée (dAdoMet): dans ces conditions la spermine continue d'être synthétisée à partir d'un pool résiduel intracellulaire de spermidine, ce qui explique dans une large mesure l'absence de réduction des concentrations cellulaires de spermine.
Figure 4. Cycle intracellulaire des polyamines (d'après [45]). ODC: ornithine décarboxylase; PAO: polyamine oxydase; cSAT: acétyICoA, spermidine/spermine N1acétyltransférase cytosolique; Spm synthase: synthase de la spermine; Spd synthase: synthase de la spermidine; dAdoMet: S-adénosylméthionine décarboxylée. processus tumoral en représente à coup sûr l'une des plus importantes. Une autre source majeure de polyamines est constituée par le tractus gastro-intestinal [6], du fait de la présence d'une microflore bactérienne en prolifération active, de l'intense renouvellement des entérocytes et de son contenu alimentaire. Certains aliments possèdent en effet des teneurs importantes en polyamines [7].

Parce qu'il est difficile de maintenir une concentration suffisante d'inhibiteur de l'ODC dans un organisme, et qu'il existe de surcroît des polyamines circulantes accessibles aux cellules cancéreuses, l'administration seule réduire de manière suffisante la quantité de polyamines tumorales, et juguler par là la progression maligne, ce que les expériences effectuées chez l'animal, comme les essais cliniques réalisés chez l'homme, ont clairement démontré.

\section{L'inhibition de la S-adénosylméthio- nine décarboxylase (AdoMetDC) est-elle porteuse de plus d'espoirs thérapeutiques?}

La transformation de la putrescine en spermidine, de même que celle de la spermidine en spermine requiert obligatoirement de la S-adénosylméthionine décarboxylée (dAdoMet) (figure 1). Mais à la différence de la putrescine qui peut, soit être synthétisée de novo à partir de l'ornithine (figure 4), soit provenir de spermidine ou de spermine extracellulaires, la dAdoMet ne peut provenir que d'une décarboxylation intracellulaire de Sadénosyl-méthionine, sous l'action de la AdoMetDC.

Dans ces conditions il était a priori permis d'espérer qu'une inhibition spécifique de l'AdoMetDC pourrait inhiber efficacement la prolifération cellulaire cancéreuse in vivo. L'administration simultanée de DFMO et de 
méthylglyoxal-bis (guanyl) hydrazone (MGBG, Méthyl-GAG ${ }^{\mathrm{TM}}$ ) (figure 2) - un médicament utilisé dans les leucémies qui se révéla secondairement être un inhibiteur compétitif de l'AdoMetDC - s'était d'ailleurs dans certains cas accompagnée d'indéniables effets anticancéreux [3], mais en règle générale associés à des effets secondaires toxiques.

L'utilisation d'un inhibiteur spécifique de l'AdoMetDC, le MDL 73.811 (figure 2), molécule comportant des analogies structurales avec la dAdoMet, a permis de démontrer que l'inhibition de cette décarboxylase était sans effet sur la prolifération cellulaire cancéreuse, alors même que cet inhibiteur effondrait les concentrations intracellulaires de spermine et de spermidine [8]. En fait, tout en diminuant les concentrations intracellulaires de spermidine et de spermine, l'inhibition de l'AdoMetDC induit corrélativement, un accroissement à la fois de l'activité et de la quantité de protéine ODC; dans ces conditions la putrescine s'accumule dans de telles proportions qu'elle peut, à elle seule, satisfaire aux besoins en polyamines d'une cellule en prolifération active. Seule une incubation suffisamment longue des cellules en présence de MDL 73.811, capable de réduire la synthèse du facteur de début de traduction IF5A, peut induire une inhibition significative de la prolifération cellulaire cancéreuse [9].

D'autres inhibiteurs de l'AdoMetDC, présentant cette fois des analogies structurales avec le MGBG, ont été récemment synthétisés: parmi ceux-ci, le CGP 48.664 (figure 2). Contrairement à l'inhibiteur précédent (MDL 73.811), le CGP 48.664 inhibe de manière significative la progressison tumorale [10], en induisant pourtant des modifications similaires des concentrations intracellulaires de polyamines.

Parmi les explications pouvant être avancées pour expliquer les effets antiprolifératifs constatés avec le CGP 48.664 (mais pas avec le MDL 73.811), l'une est que cet analogue polycationique pourrait occuper des sites intracellulaires habituellement utilisés par la spermidine et la spermine endogènes. Le CGP 48.664 pourrait ainsi mimer la structure de polyamines naturelles, sans être pour autant capable d'en assumer les fonctions biologiques.

\section{Stratégies pour une inhibition de la croissance tumorale in vivo}

Le métabolisme des polyamines est réglé de telle manière que l'inhibition isolée de l'une ou de l'autre de ses enzymes ne peut à elle seule réduire de manière suffisamment importante et durable les concentrations intracellulaires de polyamines. Toutes les tentatives allant dans le sens d'un blocage uni-enzymatique spécifique se sont à ce jour soldées par des échecs. L'inhibition couplée de l'ODC et de l'AdoMetDC ne s'est pas révélée plus efficace in vivo, les cellules cancéreuses rééquilibrant systématiquement leur pool intracellulaire de polyamines en captant les polyamines circulantes présentes dans leur environnement immédiat, en particulier dans le sang. En dépit des échecs passés, il est néanmoins actuellement permis d'envisager de nouvelles stratégies thérapeutiques faisant appel à des inhibiteurs, voire des modulateurs du métabolisme des polyamines.

\section{Inhibition de I'ODC couplée à une inhibition du transport transmembranaire des polyamines}

Il a été montré que des cellules leucémiques L1210 mutées, rendues déficientes en transporteurs transmembranaires de polyamines, étaient beaucoup plus sensibles à un traitement par la DFMO que leurs homologues non mutées [11]. Bien que nous ne disposions pas à ce jour d'un inhibiteur suffisamment puissant du transport des polyamines, les travaux en cours visant, d'une part, à la caractérisation moléculaire du transporteur protéique présent au niveau de la membrane plasmique des cellules eucaryotes et, d'autre part, à la compréhension de sa régulation qui semble très différente de celle observée chez la bactérie [12], permettent d'envisager qu'un tel traitement puisse être un jour disponible.

\section{Réduction de concentrations intracellulaires de polyamines}

Comme suggéré précédemment à propos du CGP 48.664, nous pensons que certains analogues structuraux de polyamines naturelles exercent leurs effets antitumoraux en occupant des sites d'ordinaire occupés par les polyamines naturelles; ces composés sont des analogues de la N1,N12-bis (éthyl)spermine, et répondent à la formule générale suivante [13] :

$$
\begin{gathered}
\mathrm{C}_{2} \mathrm{H}_{5}-\mathrm{NH}-\left(\mathrm{CH}_{2}\right)_{\mathbf{a}}-\mathrm{NH}-\left(\mathrm{CH}_{2}\right)_{\mathbf{b}} \\
-\mathrm{NH}-\left(\mathrm{CH}_{2}\right)_{\mathbf{c}}-\mathrm{NH}-\mathrm{CH}_{2} \mathrm{H}_{5}
\end{gathered}
$$

Du fait de leurs analogies structurales avec les polyamines naturelles, ces dérivés induisent la synthèse de l'acétylCoA:spermidine/spermine acétyltransférase (cSAT) (figure 4), et favorisent l'excrétion et la dégradation intracellulaire des polyamines acétylées [14-16]. Ces analogues de polyamines semblent aussi interagir au niveau mitochondrial [17]. Parmi eux, certains se sont révélés efficaces chez des animaux porteurs de tumeurs greffées [2, 18, 19] et sont actuellement en cours d'évaluation clinique.

Bien qu'il soit prématuré d'émettre un avis définitif concernant cette approche thérapeutique, les résultats obtenus à ce jour permettent d'être optimiste pour ce qui est de leur utilisation clinique future, et cela d'autant plus que l'activité de ces molécules est indifférente à la dépendance hormonale de ces tumeurs.

\section{Suppression in vivo des sources principales de polyamines}

La DFMO étant pratiquement dénuée d'effets toxiques chez l'homme, et cet inhibiteur spécifique de l'ODC étant de plus facile à administrer per os, le problème consistait à repenser les bases d'une stratégie thérapeutique visant à restituer à cette molécule, in vivo, les propriétés antiprolifératives qu'elle exerce in vitro. 


\section{RÉFÉRENCES}

14. Fukuchi J, Kashiwagi K, Kusama-Eguchi K, Terao K, Shirahata A, Igarashi K. Mechanism of the inhibition of cell growth by N1,N12-bis (ethyl) spermine. Eur J Biochem 1992; 209: 689-96.

15. Albanese L, Bergeron C, Pegg AE. Investigations of the mechanism by which mammalian cell growth is inhibited by N1,N12bis(ethyl) spermine. Biochem J. 1993; 291 : 131-7.

16. Fogel-Petrovich M, Shappell NW, Bergeron RJ, Porter CW. Polyamine and polyamine analog regulation of spermidine/ spermine N1-acetyltranferase (SSAT) in MALME-3M human melanoma cells. J Biol Chem 1993 ; 268 : 19118-25.

17. Vertino PM, Beerman TA, Kelly EJ, Bergeron RJ, Porter CW. Selective cellular depletion of mitochondrial DNA by the polyamine analog N1,N12-bis(ethyl) spermine and its relationship to polyamine structure and function. Mol Pharmacol 1991; 39 : 487-94.

18. Prakash NJ, Bowlin TL, Edwards ML, Sunkara P.S., Sjoerdsma A. Antitumor activity of a novel synthetic polyamine analogue, N,N'bis-(3-(ethylamino)-propyl)-1,7-heptanediamine : potentiation by polyamine oxidase inhibitors. Anticancer Res $1990 ; 10$ : 1281-8.

19. Porter CW, Bernacki RJ, Miller J, Bergeron RJ. Antitumor activity of N1,N11-bis (ethyl) norspermine against human melanoma xenografts and possible biochemical correlates of drug action. Cancer Res 1993; 53: $581-6$

20. Seiler N, Sarhan S, Grauffel C, Jones R, Knödgen B, Moulinoux JP. Endogenous and exogenous polyamines in support of tumor growth. Cancer Res 1990; $50: 5077-83$.

21. Hessels J, Kingma AW, Ferwerda H, Keij J, van den Berg GA, Muskiet FAJ. Microbial flora in the gastrointestinal tract abolishes cytostatic effets of $\alpha$-difluoromethylornithine in vivo. Int J Cancer 1989; 43: 1155-64.

22. Hessels J, Kingma AW, Muskiet FAJ, Seiler N. Growth inhibition of two solid tumors in mice, caused by polyamine depletion, is not attended by alterations in cell-cycle phase distribution. Int J Cancer 1991; 48: 697703.

23. Seiler N, Atanassov L. The natural polyamines and the imminue system. In : Jucker E, ed. Progress in drug research. Basel, Suisse: Birkhäuser 1994; 43: 87-141.

24. Chamaillard L, Quemener V, Havouis R, Moulinoux JP. Polyamine deprivation stimulates natural killer cell activity in cance-
Le tractus intestinal constituant la source prépondérante de polyamines exogènes, pour partie d'origine bactérienne et pour partie d'origine alimentaire, nous avons partiellement décontaminé le tractus intestinal des animaux par des antibiotiques (néomycine, métronidazole) et leur avons fourni un aliment de synthèse à faible teneur en polyamines. Comme le montre le Tableau I, la triple association antibiotiques intestinaux/ DFMO/aliment à faible teneur en polyamines s'est non seulement accompagnée d'un effondrement des concentrations intratumorales de putrescine et de spermidine, mais encore et surtout a réduit de manière très significative la croissance tumorale (figure 5) et la dissémination métastatique (Tableau II) [6, 20].

Bien que les entérocytes subissent la carence en polyamines infligée aux cellules tumorales, ni leur aspect histologique, ni leurs fonctions n'ont été affectés chez les animaux ainsi traités [20]. Les entérocytes ont directement accès aux polyamines présentes dans la lumière intestinale, et sont de ce fait à même de capter les quantités de polyamines nécessaires à leur métabolisme intermédiaire et à leur prolifération. L'intestin entrerait ainsi dans une manière de compétition avec le tissu tumoral en diminuant la quantité de polyamines accessible à la tumeur.

Si l'administration simultanée de DFMO et d'antibiotiques intestinaux bloque les cellules cancéreuses en phase G1 chez des animaux porteurs d'une ascite leucémique L1210 [21], aucun blocage dans une phase précise du cycle cellulaire n'a été observé en cas de tumeur solide (carcinome pulmonaire de Lewis, fibrosarcome murin, [22]). Il était alors envisageable que l'inhibition de la progression tumorale induite par une carence de l'organisme en polyamines puisse être aussi en relation avec une activation des défenses immunitaires antitumorales [23]. Nous avons ainsi constaté que l'activité cytotoxique NK, effondrée chez les animaux porteurs d'une greffe tumorale, était normalisée chez les animaux traités par la triple association antibiotiques intestinaux/DFMO/aliment à faible teneur en polyamines [24].

Dans certains cas, nous avons également inhibé l'activité de la polyamine oxydase (PAO, figure 4) en administrant aux animaux de la N1, N4-bis (2,3-butadienyl)-1,4-butanediamine (MDL 72.527); cet inactivateur de la PAO prévient en effet la rétroconversion oxydative de la spermine en spermidine, et celle de la spermidine en putrescine, ce qui a pour conséquence de réduire une autre source intracellulaire de spermidine et de putrescine.

Si l'ajout de MDL 72.527 à la triple association antibiotiques intestinaux/DFMO/aliment à faible teneur en polyamines n'a que peu d'influence sur la concentration intratumorale de polyamines (Tableau I), elle amplifie cependant de manière significati-

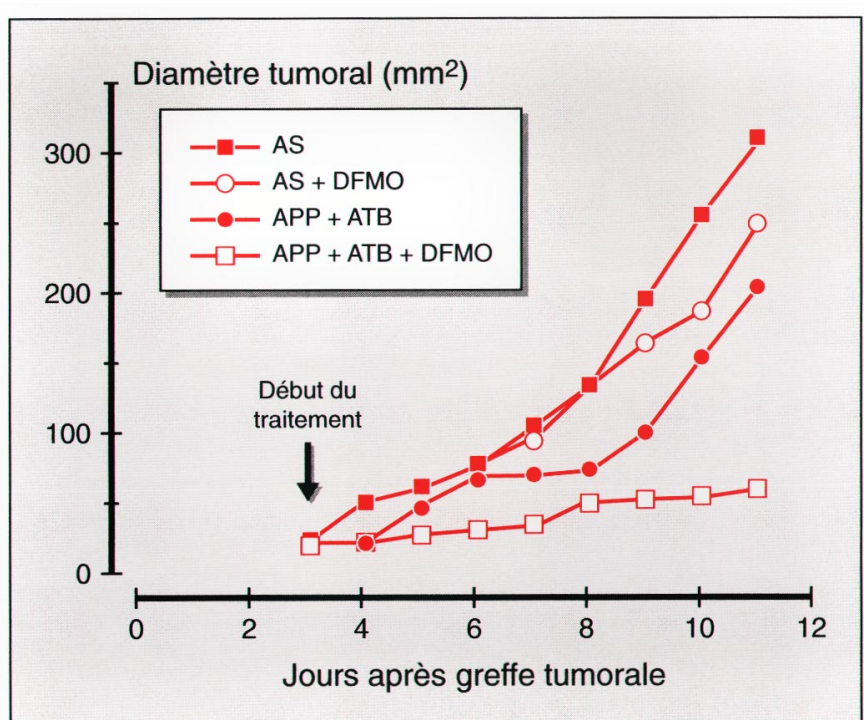

Figure 5. Effet de la carence en polyamines sur la croissance in vivo du carcinome pulmonaire de Lewis (3LL) (d'après [6]). (AS: aliment standard; APP: aliment pauvre en polyamines; $A T B$ : antibiotiques, néomyci$n e+$ métronidazole); DFMO: 2(difluorométhyl) ornithine. 
Tableau I

EFFETS DE TRAITEMENTS PROVOQUANT UNE CARENCE EN POLYAMINES (POIDS TUMORAL, TENEUR EN POLYAMINES) CHEZ DES ANIMAUX AYANT SUBI UNE GREFFE DE CARCINOME PULMONAIRE DE LEWIS 3LL

\begin{tabular}{|lccccc|}
\hline Traitement & $\begin{array}{c}\text { Poids } \\
\text { tumoral } \\
(\boldsymbol{g})\end{array}$ & Putrescine & $\begin{array}{c}\text { N1-Acétyl- } \\
\text { spermidine } \\
(\mathbf{n m o l} . \boldsymbol{g})^{-1}\end{array}$ & Spermidine & Spermine \\
\hline Aliment standard (AS) & $3,0 \pm 0,4$ & $95 \pm 16$ & $7 \pm 2$ & $956 \pm 97$ & $770 \pm 83$ \\
Aliment pauvre & $2,2 \pm 0,6$ & $103 \pm 29$ & $7 \pm 2$ & $1002 \pm 145$ & $768 \pm 145$ \\
en polyamines (APP) & & $18 \pm 10$ & $2 \pm 1$ & $226 \pm 61$ & $872 \pm 143$ \\
AS + DFMO & $2,2 \pm 0,5$ & $12 \pm 7$ & $1,3 \pm 0,3$ & $173 \pm 53$ & $577 \pm 77$ \\
APP + DFMO & $1,1 \pm 0,4$ & $11 \pm 7$ & $183 \pm 64$ & $163 \pm 54$ & $646 \pm 95$ \\
APP + DFMO & $0,2 \pm 0,1$ & & & & \\
+ MDL 72527 & & & & & \\
\hline
\end{tabular}

Aliment pauvre en polyamines (APP): aliment de synthèse constitué de précurseurs purifiés (Sigma), contenant 200 mg.100 g-1 de néomycine et $3,4 \mathrm{mg} 100 \mathrm{~g}^{1}$ de métronidazole.

La DFMO (2\%) et le MDL 72.527 (0,05\%) ont été administrés dans l'eau de boisson.

Le traitement a été mis en place en présence d'une tumeur palpable, les animaux ont été sacrifiés 11 jours après la greffe tumorale.

Données issues de [20].

Tableau II

ESTIMATION DU NOMBRE DE MÉTASTASES PULMONAIRES CHEZ DES ANIMAUX AYANT SUBI UNE GREFFE

DE CARCINOME PULMONAIRE DE LEWIS 3LL TRAITÉS PAR UNE CARENCE EN POLYAMINES

\begin{tabular}{|lcc|}
\hline Traitement & $\begin{array}{c}\text { Nombre de métastases } \\
\text { par poumon } \\
\text { (valeurs extrêmes) }\end{array}$ & $\begin{array}{c}\text { Pourcentage } \\
\text { d'animaux indemnes } \\
\text { de métastases }\end{array}$ \\
\hline Aliment standard (AS) & 14 & 0 \\
Aliment pauvre & $(4-23)$ & 0 \\
en polyamines (APP) & 1 & 0 \\
AS + DFMO & $(1-7)$ & 43 \\
APP + DFMO & 8 & 43 \\
APP + DFMO + MDL 72527 & $(2-24)$ & 43 \\
& 1 & \\
\hline
\end{tabular}

Mêmes conditions de traitements que celles exposées dans le Tableau I. Données issues de [20].

ve l'effet antitumoral du traitement (figure 5) [20]. L'inhibition de la PAO s'est d'ailleurs révélée capable de stimuler la production d'IL2 par des lymphocytes $\mathrm{T}$ activés [25], et nous avons constaté qu'il en allait de même chez des animaux cancéreux carencés en polyamines (résultats non publiés). Il se pourrait alors qu'une oxydase susceptible d'être inhibée par le MDL 72.527 joue un rôle-clé dans la régulation de fonctions dévolues aux lymphocytes $\mathrm{T}$ [23], cette molécule pouvant être alors considérée comme immunosti-
[23]. Les observations dont nous disposons actuellement concernant la stimulation des défenses immunitaires induite par une modulation du métabolisme des polyamines sont encourageantes et constituent des exemples du potentiel anticancéreux qu'il est permis d'attendre de tels traitements dans un futur proche.

\section{Association carence en polyamines - molécules cytotoxiques}

Dans un organisme tumoral carencé en polyamines, la réduction de la prolifération cellulaire cancéreuse est due en grande partie à un effet cytostatique. On ne peut donc pas envisager de réduire la masse tumorale par un tel traitement. L'association de molécules cytotoxiques à une carence en polyamines constituait donc une approche thérapeutique logique (Tableau III).

mulante. Dans le même ordre d'idée, il faut noter que l'ODC est depuis peu considérée comme une cible thérapeutique dans le cadre de la chimioprévention [26]. Actuellement la DFMO est testée chez des patients présentant un risque de développement d'un cancer du côlon [27]; les doses efficaces de DFMO prises à titre préventif sont très inférieures à celles utilisées en cas de traitement [3]. A notre avis, l'effet protecteur du DFMO vis-à-vis de la carcinogenèse est à mettre sur le compte d'une stimulation du système immunitaire
Reposant à l'origine sur l'hypothèse que la réduction des concentrations de polyamines intracellulaires pouvait faciliter l'accès de molécules alkylantes ou intercalantes à l'ADN, l'étude des effets anticancéreux de l'association DFMO/molécule cytotoxique a fait l'objet de nombreux travaux. Si l'activité anticancéreuse de médicaments telle que la cyclophosphamide, certaines nitrosourées ou encore des antimétabolites de l'ADN (cytosine arabinoside) a pu être potentialisée par l'administra- 
Tableau III

EFFETS DE LA CARENCE EN POLYAMINES ASSOCIÉE

À UNE POLYCHIMIOTHÉRAPIE SUR LA SURVIE D'ANIMAUX PORTEURS D'UNE GREFFE DE CARCINOME PULMONAIRE DE LEWIS

\begin{tabular}{|c|c|c|c|c|c|}
\hline & $\begin{array}{c}\text { AS } \\
\text { (Témoins) }\end{array}$ & $\begin{array}{c}{[A P P+A T B+D F M O]} \\
7 \mathrm{j} / \mathrm{j}\end{array}$ & $\begin{array}{c}{[A P P+A T B+D F M O]} \\
5 \mathrm{j} / 7 \\
\text { AS } \\
2 \mathrm{j} / 7\end{array}$ & $\begin{array}{c}\text { AS } \\
\stackrel{+}{\mathrm{MCV}}]\end{array}$ & $\begin{array}{c}{[A P P+A T B+I]} \\
5 \mathrm{j} / 7 \\
A S+[M C V] \\
2 \mathrm{j} / 7\end{array}$ \\
\hline $\begin{array}{l}\text { Temps } \\
\text { moyen } \\
\text { de survie } \\
\text { (jours) }\end{array}$ & 28 & 36 & 28 & 39 & 46 \\
\hline $\begin{array}{l}\text { Augmentation } \\
\text { du temps } \\
\text { de survie } \\
\text { (\%) }\end{array}$ & & 28 & 0 & 39 & 64 \\
\hline
\end{tabular}

Les animaux (8 par groupe dans tous les cas) ont reçu soit de l'aliment standard (AS), soit un aliment pauvre en polyamines (APP) contenant des antibiotiques (ATB: néomycine 0,2\%, métronidazole 0,0034\%) et de la DFMO (3\%). Selon le cas, les animaux ont été ou non traités par une polychimiothérapie i.p. (MCV: méthotrexate 1,7 mg. $\mathrm{kg}^{-1}$; cyclophosphamide $90 \mathrm{mg} \cdot \mathrm{kg}^{-1}$; vindésine $0,25 \mathrm{~kg}^{-1}$ ). Le traitement a dans tous les cas été instauré en présence d'une tumeur palpable $\left(0,2 \mathrm{~cm}^{3}\right)$.

Données issues de [30].

tion conjointe de DFMO [28, 29], d'autres médicaments comme le cisplatine ont vu, au contraire, leur activité antitumorale réduite par rapport à celle observée en l'absence de traitement par la DFMO [28]. Cela nous a conduits à envisager qu'une carence tumorale en polyamines plus poussée pouvait potentialiser les effets anticancéreux de médicaments cytotoxiques usuels. Notre hypothèse s'est révélée exacte, au moins dans le cas de tumeurs solides comme le carcinome pulmonaire de Lewis [30] (Tableau II) et l'adénocarcinome de la prostate hormono-indépendant Dunning MAT-LyLu [31] (figure 6). Ces résultats démontrent qu'il est actuellement possible d'amplifier l'efficacité de médicaments anticancéreux usuels en leur associant un traitement virtuellement non toxique, capable de réduire l'accès des polyamines à la tumeur.

\section{Polyamines et angiogenèse}

L'inhibition de l'angiogenèse tumorale représente sans nul doute une approche thérapeutique prometteuse dans le traitement des tumeurs

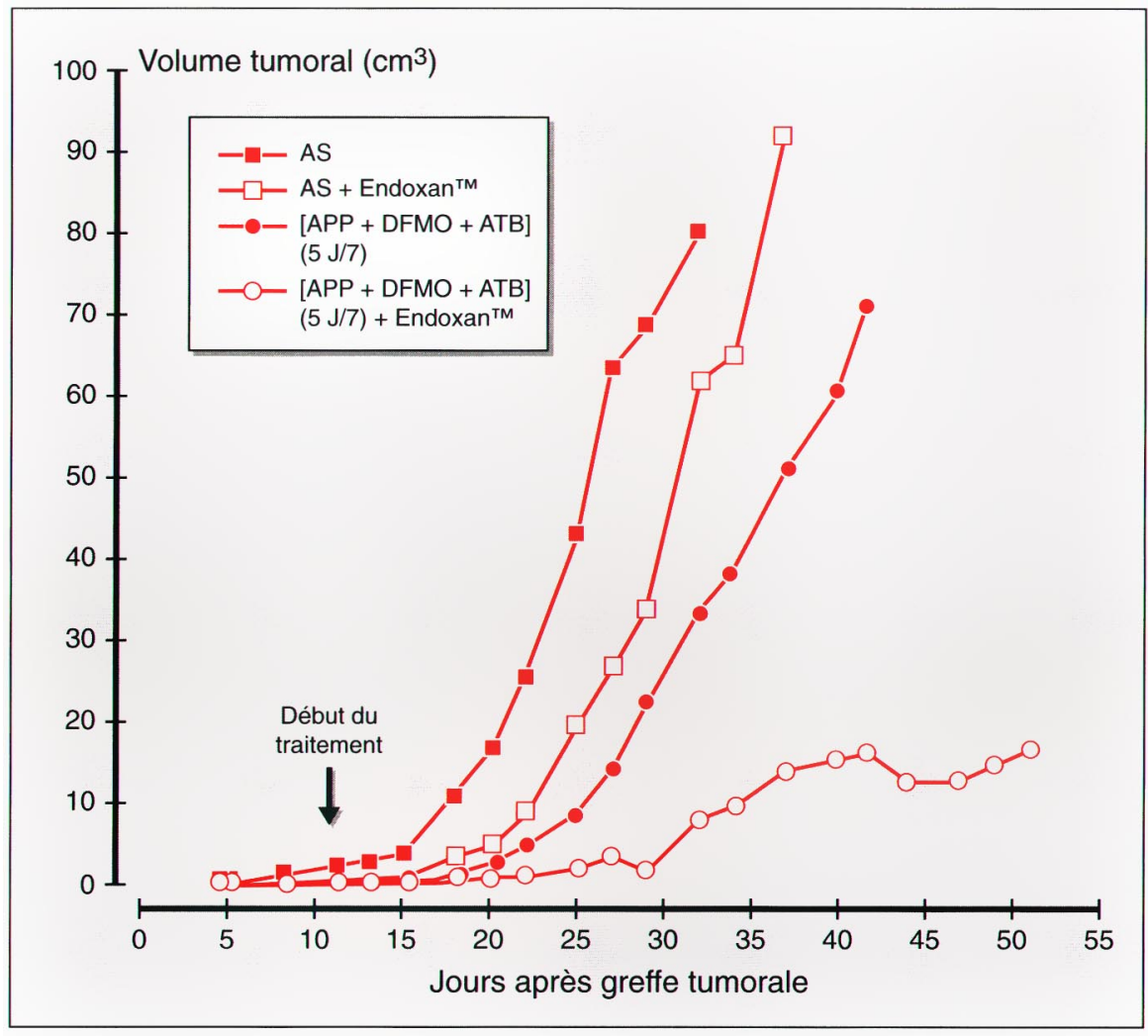

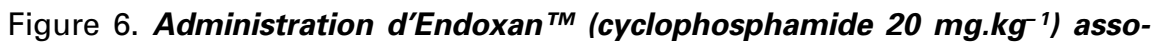
ciée à une carence partielle en polyamines (5/7 jours) sur la progression tumorale de I'adénocarcinome prostatique MAT Ly-Lu (d'après [30]). (AS: aliment standard; APP: aliment pauvre en polyamines; ATB: antibiotiques, néomycine + métronidazole). DFMO: 2-(difluorométhyl)ornithine. 


\section{RÉFÉRENCES}

25. Flescher E, Bowlin TL, Talal N. Regulation of IL-2 production by mononuclear cells from rheumatoid arthritis synovial fluids. Clin Exp Immunol 1992; 87 : 435-7.

26. Pegg AE, Shantz LM, Coleman C. Ornithine decarboxylase as a target for chemoprevention. J Cell Biochem 1995;22 (suppl) : 132-8.

27. Meyskens FL, Gerner EW. Development of difluoromethylornithine as a chemoprevention agent for the management of colon cancer. J Cell Biochem 1995; 22 (suppl) : 12631 .

28. Porter CW, Jänne J. Modulation of antineoplastic drug action by inhibitors of polyamine biosynthesis. In : McCan PP, Pegg $\mathrm{AE}$, Sjoerdsma A, eds. Inhibition of polyamine metabolism. Orlando, USA: Academic Press, 1987: 203-47.

29. Marton LJ. Effect of treatment with DNA-directed cancer chemotherapeutic agents after polyamine depletion. Pharmacol Ther 1987; 32: 183-90.

30. Quemener V, Moulinoux JP, Havouis R, Seiler N. Polyamine deprivation enhances antitumoral efficacy of chemotherapy. Anticancer Res 1992; 12: 1447-54.

31. Cipolla B, Blanchard Y, Chamaillard L, Quemener V, Guillé F, Havouis R, Moulinoux JP. In vivo, synergestic inhibition of MAT-LyLu rat prostatic adenocarcinoma growth by polyamine deprivation and low dose cyclophosphamide. Urol Res 1996 (sous presse).

32. Denekamp J. Vascular attack as a therapeutic strategy for cancer. Cancer Metastasis Rev 1990; 9: 267-82.

33. Takigawa M, Enomoto M, Nishida Y, Pan HO, Kinoshita A, Suzuki F. Tumor angiogenesis and polyamines: alpha-difluoromethylornithine, an irreversible inhibitor of ornithine decarboxylase, inhibits B16 melanoma-induced angiogenesis in ovo and the proliferation of vascular endothelial cells in vitro. Cancer Res 1990; 50: 4131-8.

34. Monte M, Klein S, Jasnis MA, Davel L, Algranati ID, Sacerdote de Lustig E. Inhibition of lymphocyte and tumor-induced angiogenesis by the administration of difluoromethylornithine. Cancer J 1993; 6 : 147-50.

35. Hull WE, Kunz W, Port RE, Seiler N. Chain-fluorinated polyamines as tumor markers III. Determination of geminal difluoropolyamines and their precursor 2,2difluoroputrescine in normal tissue and experimental tumours by in vitro and in vivo ${ }^{19} \mathrm{~F}$ NMR spectroscopy. NMR Biomed 1988; $1: 11-9$.

des polyamines dans les processus de croissance cellulaire, ces molécules sont de facto indispensables à l'angiogenèse tumorale. La DFMO provoque une réduction très significative de l'angiogenèse tumorale [33, 34]. Il est très probable que les effets antitumoraux obtenus lors de l'induction d'une carence de l'organisme en polyamines résultent au moins pour partie d'une réduction de l'angiogenèse tumorale.

\section{Vectorisation tumorale de médicaments anticancéreux par des polyamines naturelles}

A partir du moment où la tumeur est plus carencée en polyamines que les tissus sains d'un organisme traité par la DFMO, on peut envisager une autre stratégie thérapeutique qui serait de favoriser l'accumulation de molécules anticancéreuses vectorisées par des polyamines. La 2,2difluoroputrescine a été initialement utilisée dans un modèle expérimental: après injection de ce composé à des animaux porteurs d'une tumeur de Lewis recevant de la DFMO, l'accumulation maximale de difluorospermidine et de difluorospermine se produit dans le tissu tumoral et l'intestin grêle; dans ces conditions expérimentales, les autres tissus de l'organisme contiennent en effet moins de $25 \%$ de la quantité de difluoropolyamines accumulées par la tumeur [35]. Afin de favoriser l'accumulation de composés cytotoxiques par le tissu tumoral, des molécules anticancéreuses ont également été substituées par des polyamines; ce fut par exemple le cas du chlorambucil-spermidine et spermine (figure 7) [36].

Bien qu'assez récente, cette approche thérapeutique permet d'envisager de réduire dans le futur les effets secondaires toxiques de certains médicaments anticancéreux usuels. En outre, les progrès de l'imagerie médicale et de la radiologie interventionnelle permettent d'administrer des drogues à proximité immédiate de processus tumoraux d'accès chirurgical délicat, comme dans le cas des tumeurs cérébrales profondes.

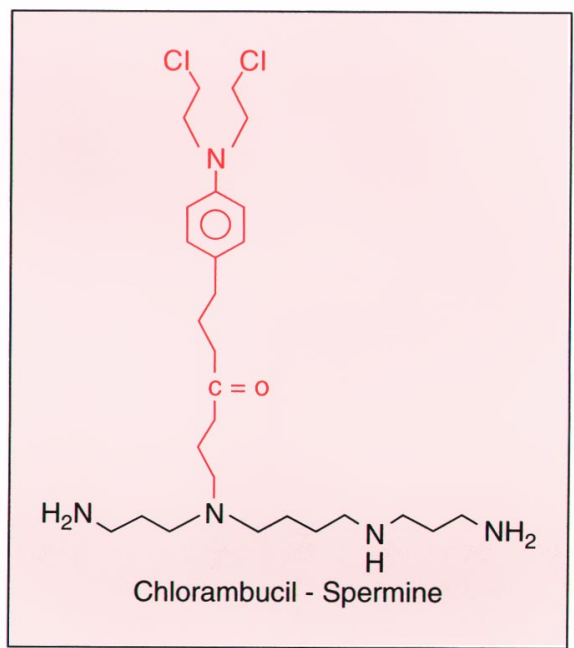

Figure 7. Chlorambucil-spermine. Les polyamines s'accumulant dans le tissu tumoral, des molécules anticancéreuses ont été substituées par des polyamines afin de favoriser l'accumulation de composés cytotoxiques par le tissu tumoral.

\section{Les polyamines en tant que marqueurs tumoraux}

Depuis les premiers travaux de Diane H. Russell [37] qui avaient pour objectif d'utiliser les concentrations urinaires de polyamines à des fins diagnostiques, de très nombreuses études ont révélé les limites de tels dosages chez les patients atteints de cancer [38]. En revanche, la quantification des concentrations de polyamines dans le LCR s'est révélée cliniquement intéressante, tout particulièrement en cas de médulloblastome [39].

Comme nous l'avons montré, la majeure partie des polyamines sanguines sont transportées par les hématies [40] et l'estimation des concentrations érythrocytaires de spermidine et de spermine constitue un outil cliniquement intéressant pour étudier les tumeurs solides à temps de doublement court. Les hématies transportant des polyamines synthétisées dans les cellules tumorales en cours de prolifération, les concentrations érytrhocytaires de polyamines reflètent dans une large mesure l'importance de la composante hyperplasique tumorale [40]; leur quantification constitue par conséquent un outil précieux pour 


\section{RÉFÉRENCES}

36. Cullis PM, Green RE, Malone ME, Merson-Davies L, Weaver R. Targeting tumor cells with polyamine - drug conjugates. Biochem Soc Trans 1994; 22: 402S.

37. Russell DH. Increased polyamine concentrations in the urine of human cancer patients. Nature 1971; 233: 144-5.

38. Bachrach U. Polyamines as markers of malignancy. In: Jucker E, ed. Progress in drug research. Basel, Swiss : Birkhäuser, 1992; 39: 9-33.

39. Marton LJ, Edwards MS, Levin VA, Lubich WP, Wilson CB. CSF polyamines: a new and important means of monitoring patients with medulloblastoma. Cancer 1981; 47 : 757-60.

40. Moulinoux JP, Quemener V, Khan NA. Biological significance of cirulating polyamines in oncology. Cell Mol Biol 1991; 37 : 773-83.

41. Behr J. L'éponge à protons : un moyen d'entrer dans une cellule auquel les virus n’ont pas pensé. médecine/sciences 1996; 12 : $56-9$.

42. Auvinen M, Paasinen A, Andersson LA, Hölttä E. Ornithine decarboxylase activity is critical for cell transformation. Nature 1992; 360 : 355-8.

43. Madhubala R, Pegg AE. Inhibition of ornithine decarboxylase and S-adenosylmethionine decarboxylase synthesis by antisense oligodeoxynucleotides. Mol Cell Biochem 1992; 118: 191-5.

44. Rudkin BB, Mamont PS, Seiler N. Decreased protein-synthetic activity is an early consequence of spermidine depletion in rat hepatoma tissue-culture cells. Biochem J 1984; 217 : 731-41.

45. Seiler N, Bolkenius FN, Rennert OM. Interconversion, catabolism and elimination of the polyamines. Med Biol 1981; 59 : discriminer les phases de progression tumorale propres à chaque patient.

La grande majorité des médicaments anticancéreux interférant avec les phases du cycle cellulaire, cela implique qu'ils ne peuvent être efficaces vis-à-vis de la progression du processus tumoral que si les cellules cibles cancéreuses sont effectivement en prolifération. L'estimation des concentrations de polyamines érythrocytaires devrait permettre de rythmer l'administration des molécules dont l'action dépend du cycle cellulaire aux phases de prolifération cancéreuse propres à chaque patient.

\section{Lipopolyamines et transfert de gènes}

et consommation d'un aliment à faible teneur en polyamines, constitue à court terme une approche digne d'intérêt en thérapeutique anticancéreuse. Ce traitement est en effet capable, non seulement de réduire la progression tumorale maligne, mais encore de potentialiser les effets antiprolifératifs de médicaments actuellement utilisés en cas de cancer. Ce traitement adjuvant est prometteur et n'a probablement pas encore livré la totalité de ses possibilités thérapeutiques.

$\mathrm{Au}$ tout début des recherches visant à utiliser le métabolisme des polyamines à des fins thérapeutiques anticancéreuses, l'idée maîtresse consistait a priori à concevoir des inhibiteurs spécifiques d'enzymes du métabolisme des polyamines, tout particulièrement de leur anabolisme. Or comme nous avons tenté de le montrer ici, la régulation de la concentration intracellulaire de polyamines s'articule avec des équilibres enzymatiques complexes et finement réglés, qui dépendent en outre de la capacité des cellules de rééquilibrer leur pool de polyamines en captant ces dernières dans leur environnement immédiat. Ces notions récentes ont provoqué un changement de stratégie. Il apparaît maintenant qu'il est préférable de concevoir des composés capables d'agir sur différents sites sensibles dévolus aux polyamines plutôt que de tenter d'inhiber telle ou telle étape du métabolisme des polyamines. Quelques analogues de ce type sont actuellement disponibles, certains d'entre eux sont en essai clinique, d'autres en cours d'étude préclinique.

En définitive, il n'existe plus d'argument en mesure d'écarter le métabolisme des polyamines du champ des recherches thérapeutiques à visée anticancéreuse, bien au contraire. Les échecs enregistrés lors des premiers essais cliniques sont pour une large part expliqués et, ce qui est beaucoup plus important, nous savons maintenant manier ce métabolisme complexe pour l'utiliser de manière rationnelle et efficace en oncologie humaine

La carence en polyamines provoquée par la triple association: inhibition de la synthèse endogène de polyamines (DFMO), décontamination du tractus intestinal (antibiotiques),

\section{TIRÉS À PART}

$\mathrm{J}$-Ph. Moulinoux. 
* ABRÉVIATIONS *

AdoMet: S-adénosylméthionine;

dAdoMet: S-adénosylméthionine décarboxylée;

AdoMetDC: S-adénosylméthionine décarboxylase;

DFMO: 2-(difluorométhyl)ornithine;

MDL 72.527: N1,N4-bis (2,3-butadienyl)-1,4-butanediamine;

MDL 73.811 : 25'-((Z)-4-amino-butenyl)méthylamino-5'-déoxyadénosi-

ne;

$O D C$ : ornithine décarboxylase;

PAO: polyamine oxydase;

cSAT: acétylCoA:spermidine/spermine N1-acétyltransférase cytosolique.

\section{Summary}

Why are polyamines of interest to cancerologists?

The designations of the natural polyamines, putrescine, spermidine and spermine signal sources of their first discovery. They have contributed considerably to the neglection of this class of biogenic amines with undoubted basic functions in cell growth and proliferation. Early clinical trials with polyamine biosynthesis inhibitors were mostly unsuccessful, as is evident now, due to the lack of understanding of how the polyamines are regulated in the vertebrate organisms. During the last decade our knowledge of polyamine metabolism has improved to the extent that the failure of the clinical trials, as well as the modest success of the attempts to use polyamines of body fluids as tumor markers, can be rationalized. It is evident now that in order to prevent tumors from growing, polyamine biosynthesis inhibition has to be combined with means suitable to prevent at least the utilization of exo- genous polyamines by the tumor. Systematic polyamine deprivation, especially in combination with the administration of well established cytotoxic drugs, improves anticancer therapy. Polyamine analogs have been synthesized which mimic some polyamine functions without, however, supporting cell growth. These new compounds promise a new approach in cancer therapy. Since polyamines are taken up by cells via specific transport systems, polyamine analogs can be rather selectively accumulated in tumors. This fact suggested the preparation of cytotoxic compounds with polyamine structure. The enhancement of selectivity and a reduced general toxicity is to be expected from this new class of drugs. Thus, there is no longer an objective argument against the use of polyamine derivatives, and biosynthesis inhibitors in anticancer therapy. 